\title{
Ein Website-Baukasten speziell für Ärzte
}

Eine Arzt-Website kann dazu beitragen, die Kommunikation zwischen Arzt und Patient zu verbessern. Doch die Mehrheit der Praxen ist gar nicht online präsent. Mit e.Praxis erleichtert Springer Medizin Ärzten den Einstieg in die Online-Welt.

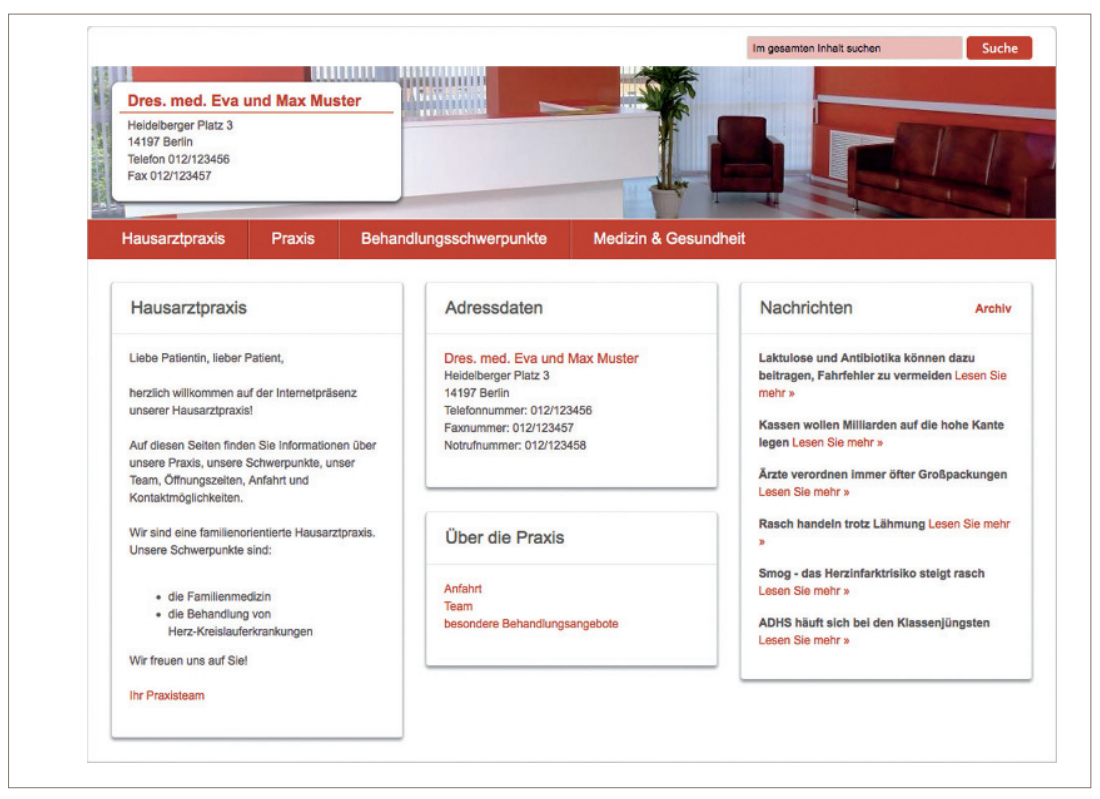

Arzt-Website aus dem Baukasten von e.Praxis

„300 Schnelltester gesucht!“ - im vergangenen Herbst startete Springer Medizin mit einem neuen Angebot, das niedergelassenen Ärzten den Weg zu einer eigenen Website erleichtern will. Bis Jahresende waren tatsächlich 300 Tester gefunden, die mit dem Homepage-Baukasten ohne Programmierkenntnisse ihre Website konstruieren und erste Erfahrungen im Internet sammeln konnten.

Die Phase des Beta-Tests ist jetzt erfolgreich abgeschlossen, die Tester konnten den Baukasten gut anwenden. Mit dem Modul e.Praxis können Ärzte nun ihre eigene Praxishomepage erstellen.

\section{Mit wenig Aufwand zu einem professionellen Webauftritt}

e.Praxis ist Teil des e.Med-Pakets von Springer Medizin, das jetzt an den Start gegangen ist. Das Modul ist vor allem für niedergelassene Ärzte, die bisher nicht im Internet vertreten sind, eine gute Gelegenheit, mit wenig Aufwand einen professionellen Webauftritt zu bekommen. Immer mehr Patienten suchen ihre Ärzte über das Internet - wer da nicht im Web präsent ist, verpasst die Chance, auch neue Patienten für seine Praxis zu gewinnen.

Der Baukasten von e.Praxis ist speziell für Ärzte entwickelt worden. Er erlaubt Ärzten ohne Program- mierkenntnisse in weniger als einer Stunde eine fertige Website zu entwickeln und mit wenig Aufwand ständig aktuell zu halten. Nutzer von e.Praxis haben die Wahl zwischen mehreren Layouts. Außerdem ist die Grundfarbe der Website frei wählbar und kann an die Farbe der Praxis angepasst werden. Basisinformationen wie Praxisöffnungszeiten und Praxisteam (inklusive Foto) lassen sich schnell zusammenstellen, aber auch Leistungsangebote der Praxis, IGeL-Angebote und ähnliches mehr sind im Baukasten schnell umgesetzt. Urlaubszeiten oder Vertretungsregelungen an Wochenenden lassen sich unkompliziert einpflegen.

\section{Medizin-News landen automatisch auf der e.Praxis-Website}

Die Websites der Testärzte, die mit dem Baukasten gearbeitet haben, können sich überwiegend sehen lassen. „Meine Diagnose: Hervorragend gelungener WebsiteBaukasten, die Bedienung macht riesig Spaß“, schreibt ein Allgemeinmediziner aus Baden Württemberg.

Einen besonders guten Eindruck machen Websites immer dann, wenn sie aktuelle Infos enthalten. Das wird bei e.Praxis dadurch gewährleistet, dass Springer Medizin täglich automatisch Medizin-News zu aktuellen Themen aus der Gesundheitspolitik auf die Arzt-Website stellt. Die Nachrichten stammen unter anderem aus der „Ärzte Zeitung“ von Springer Medizin. In Zukunft sollen Ärzte zusätzlich MedizinDossiers, zugeschnitten auf das Angebot der Praxis, auf ihre Website laden können. Die Informationen sind speziell aufbereitet für die Kommunikation für Patienten und können zum Beispiel zur Erläuterung eines Krankenbildes ausgedruckt werden.

Aber e.Praxis bietet künftig noch viel mehr: Egal ob ein Arzt sich seine personalisierte Wartezimmerzeitschrift „Wissen und Gesundheit“ dort kostenlos bestellen oder Broschüren zu einzelnen Indikationsgebieten für Patienten herunterladen möchte e.Praxis soll das Modul werden, wo niedergelassene Ärzte alles finden, was sie für eine inhaltlich richtige Patientenkommunikation über die Beratung in der Praxis hinaus benötigen. Das Beispiel EHEC hat gezeigt, wie wichtig es ist, schnell Checklisten oder fundiertes Informationsmaterial für Betroffene oder Interessierte zur Hand zu haben oder über die eigene Homepage dem Patienten zur Verfügung zu stellen.

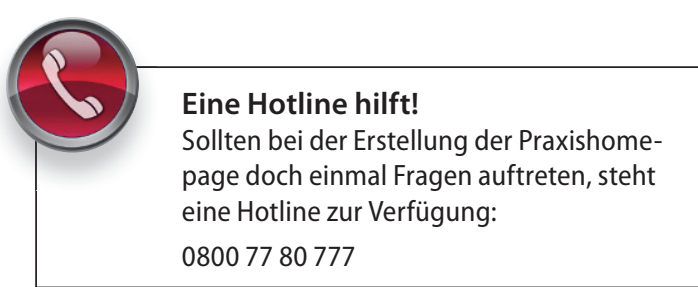

